\title{
Notes on Shape Orientation where the Standard Method Does not Work
}

\author{
Joviša Žunić*
}

Computer Science Department, Exeter University, Exeter EX4 4QF, U.K.

e-mail: J.Zunic@exeter.ac.uk

Lazar Kopanja

Department of Mathematics and Informatics, Novi Sad University

21000 Novi Sad, Trg D. Obradovića 4, Serbia and Montenegro

e-mail: Kopanjal@yahoo.com

Jonathan E. Fieldsend

Computer Science Department, Exeter University, Exeter EX4 4QF, U.K.

e-mail: J.E.Fieldsend@exeter.ac.uk

*J. Žunić is also with the Mathematical Institute, Serbian Academy of Science and Arts, Belgrade. 


\begin{abstract}
In this paper we consider some questions related to the orientation of shapes with particular attention to the situation where the standard method does not work. There are irregular and non symmetric shapes whose orientation cannot be computed in a standard way, but in the literature the most studied situations are those where the shape under consideration has more than two axes of symmetry or where it is an $n$-fold rotationally symmetric shape with $n>2$. The basic reference for our work is [11]. We give a very simple proof of the main result from [11] and suggest a modification of the proposal on how the principal axes of rotationally symmetric shapes should be computed. We show some desirable property in defining the orientation of such shapes if the modified approach is applied. Also, we give some comments on the problems that arise when computing shape elongation.
\end{abstract}

Keywords: Shape, orientation, elongation, image processing, early vision.

\title{
1 Introduction
}

The computation of a shape's orientation is a common task in the area of computer vision and image processing, being used for example to define a local frame of reference, and helpful for recognition and registration, robot manipulation, etc. It is also important in human visual perception; for instance, orientable shapes can be matched more quickly than shapes with no distinct axis [8]. Another example is the perceptual difference between a square and a diamond (rotated square) noted by Mach in 1886 [6], which can be explained by their multiple reference frames, i.e. ambiguous orientations [8].

There are situations (see Fig. 1 (a), (b), (c)) when the orientation of the shapes seems to be easily and naturally determined. On the other hand, a planar disc could be understood as a shape without orientation.

Most situations are somewhere in between. For very non-regular shapes it can be difficult to say what the orientation should be. Rotationally symmetric shapes can also have poorly defined orientation - see Fig 2 (d). Moreover, even for shapes bounded by regular polygons (see Fig. 2 (a) 


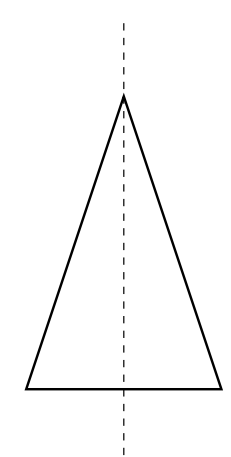

(a)

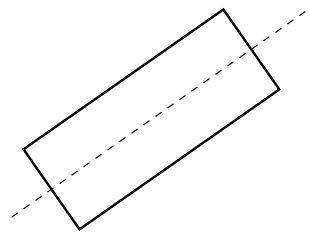

(b)

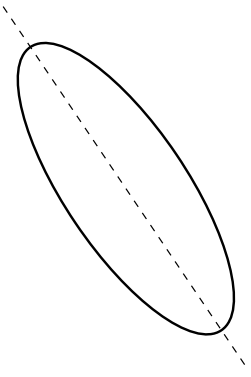

(c)

Figure 1: It is reasonable to say that the orientations of the presented shapes coincide with the dashed lines.

and (b)), it is debatable whether they are orientable or not. For instance, is a square an orientable shape? The same question arises for any regular $n$-gon, but also for shapes having several axes of symmetry, and $n$-fold $(n>2)$ rotational symmetric shapes - see shapes from Fig. 2 . It is well known $([11])$ that the standard method, based on computing the axis of the last second moment, does not suggest any answer to what the shape orientation should be if applied to $n$-fold $(n>2)$ rotationally symmetric shapes.

A compromise answer could be that such shapes are orientable but that they do not have the unique orientation. Naturally, if a $n$-fold rotationally symmetric shape is considered as an orientable shape, than it should be $n$ lines (making mutual angles that are multiplication of $2 \pi / n$ ) that define its orientation. If a shape has $n$ axes of symmetry than it is reasonable to use such axes to represent the shape orientation. Some alternative solutions are proposed in $[5,11]$.

\section{Standard Method for Computing Orientation}

In this section we give a short overview of the method which is most used in practice for computing orientation.

The standard approach defines the orientation by the so called axis of the least second moment $([1,2])$. That is, the line which minimizes the integral of the squared distances of the points 


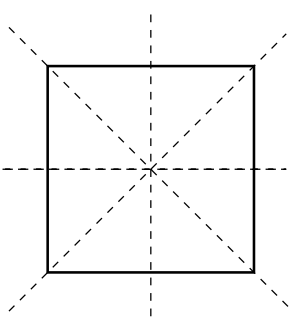

(a)

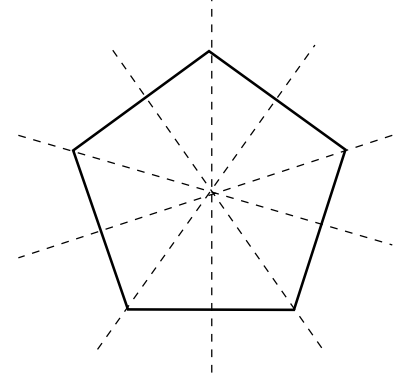

(b)

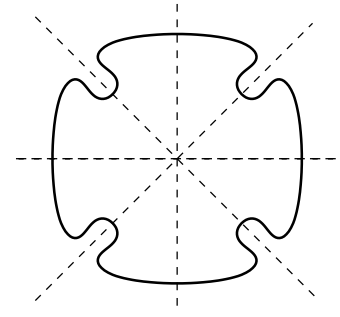

(c)

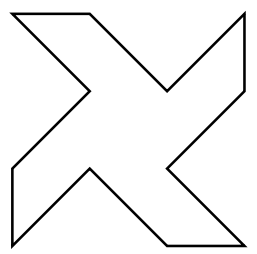

(d)

Figure 2: The dashed lines seem to be reasonable candidates to represent the orientation of the shapes (a), (b), and (c). It is not quite clear what the orientation of 4-fold rotationally symmetric shape (d) should be.

(belonging to the shape) to the line. The integral is

$$
I(\delta, \rho, S)=\iint_{S} r^{2}(x, y, \delta, \rho) d x d y
$$

where $r(x, y, \delta, \rho)$ is the perpendicular distance from the point $(x, y)$ to the line given in the form

$$
x \cdot \cos \delta-y \cdot \sin \delta=\rho .
$$

It can be shown that the line that minimizes $I(S, \delta, \rho)$ passes through the centroid $\left(x_{c}(S), y_{c}(S)\right)$ of the shape $S$ where $\left(x_{c}(S), y_{c}(S)\right)=\left(\frac{\iint_{S} x d x d y}{\iint_{S} d x d y}, \frac{\iint_{S} y d x d y}{\iint_{S} d x d y}\right)$. In other words, without loss of generality, we can assume that the origin is placed at the centroid. Since the required line minimizing $I(S, \delta, \rho)$, passes through the origin we can set $\rho=0$. In this way, the shape orientation problem can be reformulated to the problem of determining $\delta$ for which the function $I(S, \delta)$ defined as

$$
I(\delta, S)=I(\delta, \rho=0, S)=\iint_{S}(-x \cdot \sin \delta+y \cdot \cos \delta)^{2} d x d y^{1}
$$

reaches the minimum.

Further, if the central geometric moments $\bar{m}_{p, q}(S)$ are defined as usually

$$
\bar{m}_{p, q}(S)=\iint_{S}\left(x-x_{c}(S)\right)^{p} \cdot\left(y-y_{c}(S)\right)^{q} d x d y
$$

\footnotetext{
${ }^{1}$ The squared distance of a point $(x, y)$ to the line $X \cdot \cos \delta-Y \cdot \sin \delta=0$ is $(-x \sin \delta+y \cos \delta)^{2}$.
} 
and by the assumed equality $\left(x_{c}(S), y_{c}(S)\right)=(0,0)$, we obtain

$$
I(\delta, S)=(\sin \delta)^{2} \cdot \bar{m}_{2,0}(S)-\sin (2 \cdot \delta) \cdot \bar{m}_{1,1,}(S)+(\cos \delta)^{2} \cdot \bar{m}_{0,2}(S)
$$

The minimum of the function $I(\delta, S)$ can be computed easily. Setting the first derivative $I^{\prime}(x, S)$ to zero, we have

$$
I^{\prime}(\delta, S)=\sin (2 \delta) \cdot\left(\bar{m}_{2,0}(S)-\bar{m}_{0,2}(S)\right)-2 \cdot \cos (2 \delta) \cdot \bar{m}_{1,1}(S)=0 .
$$

That easily gives that the required angle $\delta$, but also the angle $\delta+\pi / 2$, satisfies the equation

$$
\frac{\sin (2 \delta)}{\cos (2 \delta)}=\frac{2 \cdot \bar{m}_{1,1}(S)}{\bar{m}_{2,0}(S)-\bar{m}_{0,2}(S)}
$$

Consequently, the maximum and minimum of $I(\delta, S)$ are as follows

$$
\begin{aligned}
& \max \{I(\delta, S) \mid \delta \in[0,2 \pi]\}=\frac{\bar{m}_{2,0}(S)+\bar{m}_{0,2}(S)+\sqrt{4 \cdot \bar{m}_{1,1}(S)+\left(\bar{m}_{2,0}(S)-\bar{m}_{0,2}(S)\right)^{2}}}{2} \\
& \min \{I(\delta, S) \mid \delta \in[0, \pi]\}=\frac{\bar{m}_{2,0}(S)+\bar{m}_{0,2}(S)-\sqrt{4 \cdot \bar{m}_{1,1}(S)+\left(\bar{m}_{2,0}(S)-\bar{m}_{0,2}(S)\right)^{2}}}{2}
\end{aligned}
$$

Let us mention that, when working with digital objects which are actually digitizations of real shapes, then central geometric moments $\bar{m}_{p, q}(S)$ are replaced with their discrete analogue, i.e., with so called central discrete moments. Since the digitization on the integer grid $\mathbf{Z}^{2}$ of a real shape $S$ consists of all integer points inside $S$ it is natural to approximate $\bar{m}_{p, q}(S)$ by the central discrete moment $M_{p, q}(S)$ defined as

$$
M_{p, q}(S)=\sum_{(i, j) \in S \cap \mathbf{Z}^{2}}\left(i-x_{c d}(S)\right)^{p} \cdot\left(j-y_{c d}(S)\right)^{q}
$$

$\left(x_{c d}(S), y_{c d}(S)\right)=\left(\frac{\sum_{(x, y) \in S \cap \mathbf{Z}^{2}} x}{\sum_{(x, y) \in S \cap \mathbf{Z}^{2}} 1}, \frac{\sum_{(x, y) \in S \cap \mathbf{Z}^{2}} y}{\sum_{(x, y) \in S \cap \mathbf{Z}^{2}} 1}\right)$ is the centroid of discrete shape $S \cap \mathbf{Z}^{2}$.

Some details about the efficiency of the approximation $\bar{m}_{p, q}(S) \approx M_{p, q}(S)$ can be found in [3].

If all the geometric moments in (3) are replaced with their corresponding discrete moments we have the equation

$$
\frac{\sin (2 \delta)}{\cos (2 \delta)}=\frac{2 \cdot M_{1,1}(S)}{M_{2,0}(S)-M_{0,2}(S)}
$$

which describes the angle $\delta$ which is used to describe the orientation of discrete shape $S \cap \mathbf{Z}^{2}$. It is worth noting that equation (5) can be derived easily if the orientation of the discrete set (a 
finite number point set) $S \cap \mathbf{Z}^{2}$ is defined by the line (passing the origin) which minimizes the sum $\sum_{(i, j) \in S \cap \mathbf{Z}^{2}}(i \cdot \sin \delta-j \cdot \cos \delta)^{2}$ of squared distances of points from $S \cap \mathbf{Z}^{2}$ to this line. In other words, the equality (5) is obtained when try to solve the following optimization problem

$$
\min \left\{I(\delta, S)=\sum_{(i, j) \in S \cap \mathbf{Z}^{2}}(i \cdot \sin \delta-j \cdot \cos \delta)^{2} \mid \delta \in[0, \pi]\right\}
$$

assuming that the centroid $\left(x_{c d}\left(S \cap \mathbf{Z}^{2}\right), y_{c d}\left(S \cap \mathbf{Z}^{2}\right)\right)$ coincides with the origin.

So, the standard method is very simple (in both "real" and "discrete" versions) and it comes from a natural definition of the shape orientation. However, it is not always effective. Indeed, if $I(\delta, S)$ is a constant function then the standard method does not work at all. I.e. the method does not tell us what the angle should be that is used to define the orientation of $S$. The equality $I(\delta, S)=$ constant could be satisfied for very non regular shapes, but it is guaranteed for all shapes that have more than two axes of symmetry, and more generally, for $n$-fold rotationally symmetric shapes for $n>2$.

The next lemma (it is a particular case of Theorem 1 from [11]) shows easily that the standard method cannot be used to orient a shape that has more than two axes of symmetry.

Lemma 1 If a given shape has more than two axes of symmetry then $I(\delta, S)$ is a constant function.

Proof. From (2) it is obvious that $I(\delta, S)$ can have no more than one maximum and one minimum on the interval $[0, \pi)$ if it is not a constant function. Trivially $I(0, S)=I(\pi, S)$. So, if $S$ has more than two axes of symmetry $I(\delta, S)$ must be constant since a non constant first derivative $I^{\prime}(\delta, S)$ could not have more than two zeros on the interval $[0, \pi)$.

Remark 1 Lemma 1 implies that $I(S, \delta)=\frac{1}{2} \cdot\left(\bar{m}_{2,0}(S)+\bar{m}_{0,2}(S)\right) \quad($ for all $\delta \in[0,2 \pi)$ ) if $S$ has more than two axes of symmetry and the standard method cannot be used to compute the orientation of $S$. The method is limited by the simplicity of the function $I(\delta, S)$ and it is useless in such situations.

\section{High-Order Principal Axes}

Rotationally symmetric shapes are such shapes which are identical to themselves after being rotated through any multiple of $2 \pi / n$. So, if a discrete point set $S$ is $n$-fold rotationally symmetric 
then it is of the form

$$
S=\left\{\left(r_{i}, \theta_{i, j}\right) \mid i=1,2, \ldots, m, j=1,2, \ldots, n \text { and } \theta_{i, j}=\theta_{i, 1}+(j-1) \frac{2 \pi}{n}\right\}
$$

where points $\left(r_{i}, \theta_{i, j}\right)$ from $S$ are given in polar coordinates. As noted before, since the function $I(\delta, S)$ has not a big enough number of local maxima and minima it cannot be used to define the orientation of $n$-fold ( $n>2$ ) rotationally symmetric shapes. In order to overcome such a problem, the authors of [11] proposed the use of $N^{t h}$-order central moments of inertia. A precise definition follows.

Definition 1 Let a shape $S$ whose centroid coincide with the origin. Then, the $N$-order central moment of inertia, denoted as $I_{N}(\delta, S)$ about a line going through the shape centroid with slope $\tan \delta$ is defined as

$$
I_{N}(\delta, S)=\sum_{(x, y) \in S}(-x \sin \delta+y \cos \delta)^{N}
$$

In order words, [11] suggests that a more complex function than (2) should be used. Obviously, if $N=2$ we have the standard method.

A nice result, related to $n$-fold rotationally symmetric shapes and their corresponded $N^{t h}$-order central moments has been proved in [11]. The proof presented there uses basic mathematics but it is pretty long (it involves many trigonometric identities). Here, we give a very elementary proof.

Theorem 1 ([11]) For an n-fold rotationally symmetric shape S, having the centroid coincident with the origin, its $N^{\text {th }}$-order central moment of inertia $I_{N}(\delta, S)$ is constant about any line going through its centroid for all $N$ less than $n$.

Proof. Let $S$ be an $n$-fold rotationally symmetric shape, with the centroid placed at the origin. First, we will derive that there are not more than $2 N$ values of $\delta$ for which $\frac{d I_{N}(\delta, S)}{d \delta}$ vanishes. Indeed, starting from

$$
\frac{d I_{N}(\delta, S)}{d \delta}=\sum_{(x, y) \in S} N \cdot(-x \sin \delta+y \cos \delta)^{N-1} \cdot(-x \cos \delta-y \sin \delta)
$$

we distinguish two situations (denoted by (i) and (ii)) depending on the values of $\frac{d I_{N}(\delta=0, S)}{d \delta}$ and $\frac{d I_{N}(\delta=\pi, S)}{d \delta}$ 
(i) - If $\delta=0$ and $\delta=\pi$ (i.e. $\sin \delta=0$ ) are not solution of $\frac{d I_{N}(\delta, S)}{d \delta}=0$, then (from (9))

$$
\frac{d I_{N}(\delta, S)}{d \delta}=0 \quad \Leftrightarrow \quad(\sin \delta)^{N} \cdot \sum_{(x, y) \in S}(-x+y \cot \delta)^{N-1} \cdot(x \cot \delta+y)=0 .
$$

Since the quantity

$$
\sum_{(x, y) \in S}(-x+y \cot \delta)^{N-1} \cdot(x \cot \delta+y)
$$

is an $N$-degree polynomial on $\cot \delta$ it cannot have more than $N$ real zeros

$$
\cot \delta_{1}=z_{1}, \quad \cot \delta_{2}=z_{2}, \quad \ldots, \quad \cot \delta_{k}=z_{k}, \quad(k \leq N)
$$

In other words, because of $\cot \delta=\cot (\delta+\pi)$ the equation

$$
\frac{d I_{N}(\delta, S)}{d \delta}=0
$$

has no more than $2 N$ solutions, if $I_{N}(\delta, S)$ is not a constant function.

(ii) - If $\delta=0$ and $\delta=\pi$ (i.e. $\sin \delta=0$ ) are solution of $\frac{d I_{N}(\delta, S)}{d \delta}=0$, than easily (see (9))

$$
\sum_{(x, y) \in S} x \cdot y^{N-1}=0
$$

But, in such a situation

$$
\sum_{(x, y) \in S}(-x+y \cot \delta)^{N-1} \cdot(x \cot \delta+y)
$$

is an $(N-1)$-degree polynomial on $\cot \delta$ (because of (10) coefficient of $(\cot \delta)^{N}$ vanishes) and consequently, it cannot have more than $N-1$ real zeros:

$$
\cot \delta_{1}=z_{1}, \quad \cot \delta_{2}=z_{2}, \quad \ldots, \quad \cot \delta_{k}=z_{k}, \quad(k \leq N-1),
$$

i.e. there are no more $2(N-1)$ values of $\delta$ for which $\sum_{(x, y) \in S}(-x+y \cot \delta)^{N-1} \cdot(x \cot \delta+y)$ vanishes. So, again, $\frac{d I_{N}(\delta, S)}{d \delta}=0$ has no more $2 N$ solutions, including $\delta=0$ and $\delta=\pi$.

Thus, in both cases the number of zeros of $\frac{d I_{N}(\delta, S)}{d \delta}$ is upper bounded by $2 N$.

On the other side, if $S$ is a fixed $n$-fold rotationally symmetric shape, then $I_{N}(\delta, S)$ must have (because of the symmetry) at least $n$ local minima and $n$ local maxima (one minimum and one maximum at any interval of the form $[\beta, \beta+2 \pi / n)$, or it must be a constant function. That means, $\frac{d I_{N}(\delta, S)}{d \delta}$ must have (at least) $2 n$ zeros $\delta_{1}, \delta_{2}, \ldots, \delta_{2 n}$.

Since the presumption $N<n$ does not allow $2 n$ zeros of $\frac{I_{N}(\delta, S)}{d \delta}$ if $I_{N}(\delta, S)$ is not a constant function, we just derived a contradiction. So, $I_{N}(\delta, S)$ must be constant for all $N$ less than $n$. 


\section{Comments on High Order Principle Axes}

Computing the orientation is not always easy and straightforward. As shown by Lemma 1, even the orientation of a square cannot be computed if the standard method is applied. Once again, the standard method, if it works, gives only one line which should represent the shape orientation. Lemma 1 is related to shapes having more than two axes of symmetry but there are also irregular shapes whose orientation is not computable by the standard method. Since it is clear that the function (2) (that uses the second degree moments only) is not powerful enough to define the orientation of any shape, [11] involves more complex functions $I_{N}(\delta, S)$ that should be used to define the orientation of $n$-fold rotationally symmetric shapes. Precisely, [11] defines an $N$-th order principal axis of a degenerate shape $S$ (a shape for which the standard method does not work) as a line going through the centroid of $S$ about which the $I_{N}(\delta, S)$ is minimized. Then, the orientation of $S$ is defined by one of $N$-th order principal axes. Of course, for any fixed $N$ there are still shapes whose orientation cannot be computed in this generalized manner - it is enough to consider an $n$-fold rotationally symmetric shape with $n>N$ (see Theorem 1 ).

Theorem 1 gives a clear answer that for an $n$-fold rotationally symmetric shape, the $N$-th order principal axes cannot be determined for all $N<n$. On the other hand, even Theorem 1 says nothing about the existence of minima (maxima) of $I_{N}(\delta, S)$ it seems that the order equal to $n$ could be an appropriate choice used to define the high order principal axes for such a shape. If $n$-th order principal axes of an $n$-fold rotationally symmetric shape $S$ exist, then they can be computed easily, as given by the next lemma.

Lemma 2 ([11]) The directions, $\delta$, of the $N^{\text {th }}$-order principal axes of an $n$-fold rotationally symmetric S satisfy following equations:

$$
\tan (n \delta)=\left\{\begin{array}{cc}
\frac{n \cdot M_{n-1,1}(S)}{M_{n, 0}(S)-(n-1) \cdot M_{n-2,2}(S)} & \text { if } n \text { is even } \\
\frac{-M_{n, 0}(S)}{M_{n-1,1}(S)} & \text { if } n \text { is odd. }
\end{array}\right.
$$

Remark 2 It is important to notice that Lemma 2 does say nothing if $S$ is not $n$-fold rotationally symmetric. 
Some examples of shape orientations obtained by a use of higher order principal axes are given Fig. (3). In the presented cases, the method satisfies the basic request for which it was involved - i.e. it suggests a precise answer to what the orientation of $n$-fold symmetric shapes should be. That could be enough for, lets say, image normalization tasks. Also, a very nice property is given by Lemma 2 - i.e. in the case when $S$ is an $n$-fold rotationally symmetric shape (with a known $n$ ) then the computation of principal axes is very simple.

On the other hand, looking at the presented examples, we can see that sometimes (in the even case) the principal axes coincide with the symmetry axes, but sometimes (in the odd case) not. This can be a strong objection because the preference that the shape orientation coincides with one of its symmetry axes (if any) seems to be reasonable. This disadvantage is caused by the fact that there is not a good enough "geometric" motivation to use centralized geometric moments having an odd order. We give two arguments for such a judgement.

The first argument comes from an easy consequence of (8):

$$
I_{2 k+1}(\delta, S)=-I_{2 k+1}(\delta+\pi, S) .
$$

Thus, if the minimum $(-1) \cdot I_{2 k+1}\left(\delta_{0}, S\right)$ is reached for an angle $\delta_{0}$ then the maximum is $I_{2 k+1}\left(\delta_{0}+\right.$ $\pi, S)$ and it is reached for the angle $\delta_{0}+\pi$. That means that the same line has to be used to describe the orientation either is measured as the minimum or maximum of $I_{2 k+1}(\delta, S)$.

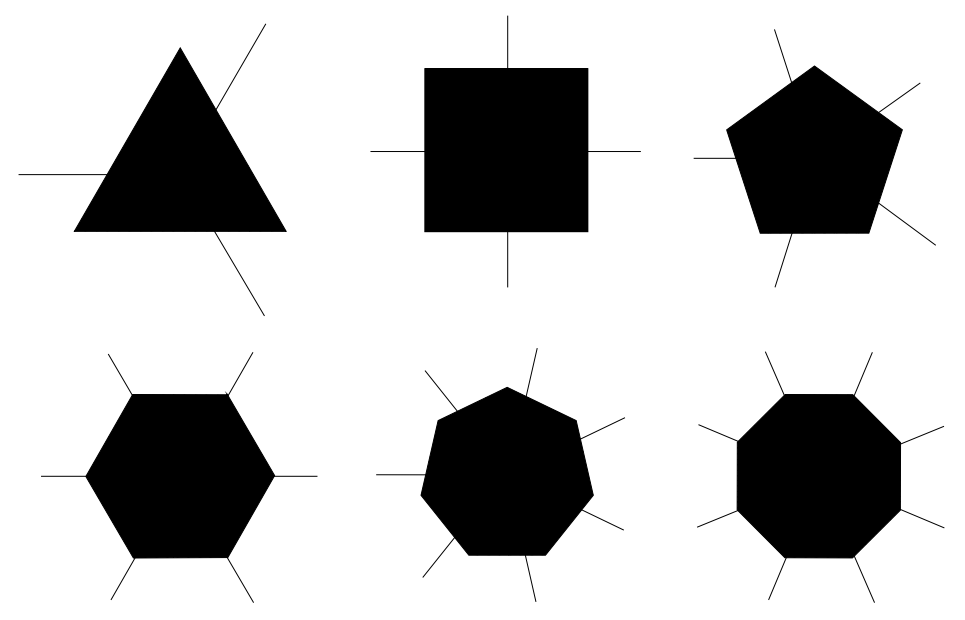

Figure 3: The principal axes (obtained as suggested in [11]) for regular 3, 4, 5, 6, 7, and 8-gons are presented. 
The second argument is based on the equality (a proof is given below)

$$
\frac{d I_{2 k+1}(0, S)}{d \delta}=-(2 k+1) M_{1,2 k}(S)
$$

that holds for the shapes positioned such that their axis of symmetry coincide with the $x$-axis. Thus, if a shape $S$ has at least one symmetry axis and if the orientation is computed as the line that minimizes $I_{2 k+1}(\delta, S)$ then it is very likely that such a line will not coincide with any axis of symmetry of $S$.

We prove (11) under the assumption that $S$ is an axis-symmetric set whose axis of symmetry coincides with the $x$-axis. So, $S$ is the union of the following sets:

- set $A$ consisting of all points from $S$ that have a positive $y$ coordinate,

- set $B$ consisting of all points from $S$ that have a negative $y$ coordinate, and

- set $C$ consisting of all points from $S$ that have $y$ coordinate equal to zero.

Since $S$ is positioned such that the $x$-axis is a symmetry axis of $S$, we have $(x, y) \in A \Leftrightarrow$ $(x,-y) \in B$. Thus, we can write:

$$
\begin{aligned}
I_{2 k+1}(\delta, S)= & \sum_{(x, y) \in A}(-x \sin \delta+y \cos \delta)^{2 k+1}+\sum_{(x, y) \in B}(-x \sin \delta+y \cos \delta)^{2 k+1} \\
& +\sum_{(x, y) \in C}(-x \sin \delta+y \cos \delta)^{2 k+1}= \\
= & \sum_{(x, y) \in A}\left((-x \sin \delta+y \cos \delta)^{2 k+1}+(-x \sin \delta-y \cos \delta)^{2 k+1}\right) \\
& +\sum_{(x, 0) \in C}(-x \sin \delta)^{2 k+1} .
\end{aligned}
$$

The first derivative is

$$
\begin{aligned}
\frac{d I_{2 k+1}(\delta, S)}{d \delta} & =\sum_{(x, y) \in A}(2 k+1) \cdot(-x \sin \delta+y \cos \delta)^{2 k} \cdot(-x \cos \delta-y \sin \delta) \\
& +\sum_{(x, y) \in A}(2 k+1) \cdot(-x \sin \delta-y \cos \delta)^{2 k} \cdot(-x \cos \delta+y \sin \delta) \\
& +\sum_{(x, 0) \in C}(2 k+1) \cdot(-x \sin \delta)^{2 k}(-x \cos \delta) .
\end{aligned}
$$


From the last equality we obtain

$$
\frac{d I_{2 k+1}(0, S)}{d \delta}=-(4 k+2) \sum_{(x, y) \in A} x y^{2 k}=-(2 k+1) M_{1,2 k}(S) .
$$

Again, $d I_{2 k+1}(0, S) / d \delta$ is not necessarily equal to zero and, consequently, a maximum is not guaranteed.

Remark 3 It is interesting to note that $d I_{2 k+1}(\pi / 2, S) / d \delta=0$ holds for any shape $S$ having a symmetry axis coincident with the $x$-axis.

So, if shape $S$ is in a general position and if $I_{2 k+1}(\delta, S)$ reaches the maximum for an angle value $\delta_{0}$, (i.e. the minimum is $-I_{2 k+1}\left(\pi+\delta_{0}, S\right)$ ) then it seems to be more reasonable to define the orientation of $S$ by the angle $\pi / 2+\delta_{0}$ (rather than by the angle $\delta_{0}$, as suggested by [11]) because it is very likely that the symmetry axis of $S$ makes an angle of $\pi / 2+\delta_{0}$ with the $x$-axis.

\section{Modified Use of High Order Principal Axes}

Here, we suggest a modified approach to the problem. We have to accept that a use of a more complex method than the standard one is necessary. So, we are going to use $N^{t h}$-order central moments with $N>2$ and will try to make a compromise between the following requests:

(c1) The method should have a reasonable geometric motivation;

(c2) The method should give some answer what orientation should be even for rotationally symmetric shapes;

(c3) The method should give reasonably good results if applied to non regular shapes;

(c4) The orientation should be relatively easy to compute.

If we go back to the standard definition of shape orientation, we can see that it is defined by the line that minimizes the sum of squares of distances of the points to this line. The squared distance (rather than the pure Euclidean distance) has been taken in order to enable an easy mathematical calculation. Following this initial idea and taking into account the problems explained by Theorem 1, we suggest that the orientation should be defined as a line which minimizes the total sum of a (suitably chosen) even-power of distances of the points to the line. We give a formal definition. 
Definition 2 Let $k$ be a given integer and let $S$ be a given shape whose centroid coincide with the origin. Then the orientation of $S$ is defined by an angle $\delta$ that minimizes

$$
I_{2 k}(\delta, S)=\sum_{(x, y) \in S}(-x \sin \delta+y \cos \delta)^{2 k} .
$$

Now, we show a desirable property of the orientation computed in accordance with the above definition. Let $S$ be an axis-symmetric set. Without loss of generality we can assume that the axis of symmetry of $S$ coincides with the $x$-axis. Again, as in the case $N=2 k+1$, we represent $S$ as the union of the sets:

- set $A$ consisting of all points from $S$ that have a positive $y$ coordinate,

- set $B$ consisting of all points from $S$ that have a negative $y$ coordinate, and

- set $C$ consisting of all points from $S$ that have $y$ coordinate equal to zero.

Since the $x$-axis is a symmetry axis of $S$, we have $(x, y) \in A \Leftrightarrow(x,-y) \in B$. Thus, we can write:

$$
\begin{aligned}
I_{2 k}(\delta, S)= & \sum_{(x, y) \in A}(-x \sin \delta+y \cos \delta)^{2 k}+\sum_{(x, y) \in B}(-x \sin \delta+y \cos \delta)^{2 k} \\
& +\sum_{(x, y) \in C}(-x \sin \delta+y \cos \delta)^{2 k} \\
= & \sum_{(x, y) \in A}\left((-x \sin \delta+y \cos \delta)^{2 k}+(-x \sin \delta-y \cos \delta)^{2 k}\right) \\
& +\sum_{(x, 0) \in C} 2 k(-x \sin \delta)^{2 k}
\end{aligned}
$$

and for the first derivative:

$$
\begin{aligned}
\frac{d I_{2 k}(\delta, S)}{d \delta} & =\sum_{(x, y) \in A} 2 k(-x \sin \delta+y \cos \delta)^{2 k-1}(-x \cos \delta-y \sin \delta) \\
& +\sum_{(x, y) \in A} 2 k(-x \sin \delta-y \cos \delta)^{2 k-1}(-x \cos \delta+y \sin \delta) \\
& +\sum_{(x, 0) \in C} 2 k(-x \sin \delta)^{2 k-1}(-x \cos \delta) .
\end{aligned}
$$

From the last equality we see that the first derivative of $I_{2 k}$ vanishes if $\delta=0$, but also if $\delta=\pi / 2$, i.e.

$$
\frac{d I_{2 k}(0, S)}{d \delta}=\frac{d I_{2 k}(\pi / 2, S)}{d \delta}=0
$$


The above equality shows that a symmetry axis (if any) has a "good chance" to be coincident with the computed orientation if Definition 2 is applied.

Since naturally defined, the orientation computed in proposed manner should performs well if applied to non regular shapes - that is illustrated by a few examples on Fig. 5-7.

Of course, the main disadvantage of the modified method is a higher computation complexity caused by the degree $2 k$ of the used central moment. It is not expected that a closed formula (as it is the formula (3) in the case of $2 k=2$ ) could be derived, but $I_{N}(\delta, S)$ written in the form

$$
\begin{gathered}
I_{N}(\delta, S)=\sum_{(x, y) \in S}(-x \sin \delta+y \cos \delta)^{N}=\sum_{i=0}^{N}\left(\begin{array}{c}
N \\
i
\end{array}\right)(-1)^{N-i}(\sin \delta)^{N-i}(\cos \delta)^{i} M_{N-i, i}(S) \\
=(-1)^{N}(\sin \delta)^{N} M_{N, 0}(S)+(-1)^{N-1} N(\sin \delta)^{N-1}(\cos \delta) M_{N-1,1}(S)+\ldots+(\cos \delta)^{N} M_{0, N}(S)
\end{gathered}
$$

enables an easy and straightforward numerical computation.

\section{Some Examples}

In this section we give some examples in order to illustrate the previous discussion. The orientation of the presented shapes is computed by minimizing $I_{N}$ for $N=2,4,6,8$, and 10 .

We start with $n$-fold rotationally symmetric shapes from Fig. 4. Since we have used digitized images the presented shapes are not perfectly symmetric. There is an inherent error caused by the digitization ("rounding") process. The obtained results are in accordance with theoretical observations. Considering the ratio (see Table 5) between the minimum and maximum of $I_{N}(\delta, S)$ we can see that $I_{2}(\delta, F i g .4(a)), I_{4}(\delta, F i g .4(a)), I_{2}(\delta, F i g .4(b)), I_{4}(\delta, F i g .4(b))$, $I_{6}(\delta, F i g .4(b)), I_{8}(\delta, F i g .4(b)), I_{2}(\delta, F i g .4(c)), I_{2}(\delta, F i g .4(d)), I_{4}(\delta, F i g .4(d)), I_{6}(\delta, F i g .4(d))$, and $I_{8}(\delta, F i g .4(d))$ are nearly constant functions (in accordance with Theorem 1). From the obtained results, taking into account the existence of the digitization error, it is some uncertainty in deciding is it $I_{10}(\delta, F i g 4 .(b))$ constant or not. An explanation can be that a regular 5-gon is very similar to the circle and consequently it has very poor orientation. The problem can be solved by using $I_{N}$ with a bigger degree. Indeed, the ratio of minimum and maximum of $I_{20}(\delta, F i g .4(b))$ is computed as 1.2732 and it is reached for $60^{\circ}$ as expected. It is important to note that the obtained results say that the orientation of shapes on Fig.4(a), Fig.4(b), and Fig.4(d), that have an odd 
number of axes of symmetry, is coincident with one of their symmetry axes. It does not happen if the method given by [11] is applied - see Fig. 3 .

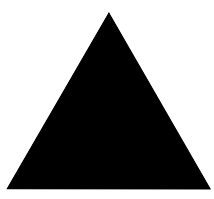

(a)

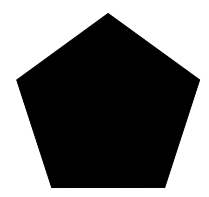

(b)

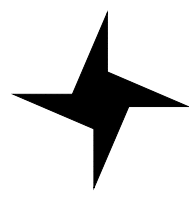

(c)

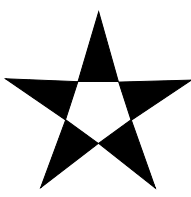

$(\mathbf{d})$

Figure 4: Rotationally symmetric shapes.

Table 1: Orientations as computed by applying Definition 2 to the shapes form Fig. 4. " - - - -" means that $I_{N}(\delta, S)$ is estimated as constant functions (i.e. the orientation cannot be computed).

\begin{tabular}{lcccc}
\hline \hline & Fig. 4(a) & Fig. 4(b) & Fig. 4(c) & Fig. 4(d) \\
\hline$N=2$ & ---- & ---- & ---- & ---- \\
\hline$N=4$ & ---- & ---- & $\approx 44^{\circ}$ & ---- \\
\hline$N=6$ & $\approx 150^{\circ}$ & ---- & $\approx 43^{\circ}$ & ---- \\
\hline$N=8$ & $\approx 150^{\circ}$ & ---- & $\approx 42^{\circ}$ & ---- \\
\hline$N=10$ & $\approx 150^{\circ}$ & $\approx 90^{\circ}$ & $\approx 42^{\circ}$ & $\approx 90^{\circ}$ \\
\hline \hline
\end{tabular}

Next, we consider the shapes that have exactly one symmetry axis. Such shapes are expected to be easily orientable for arbitrary degree of the used central moment of inertia. In the case of Fig.5(a), Fig.5(b), and Fig.5(c) the computed minimal values for $I_{2}, I_{4}, I_{6}, I_{8}$, and $I_{10}$ are obtained for an angle of 90 degrees. In the case of Fig.5(d) the computed orientation slightly varies around $92^{\circ}$. That can be explained with a much bigger digitization effects (than those in the previous three cases) that made the figure "slightly" non symmetric (not that the shape on Fig.5(d) is represented with black pixels only). The related results given in Table 5 also indicate that the shapes from Fig. 5 have a well defined orientation.

Fig. 6 displays shapes that could be understood as shapes with not well defined orientation. So, as it is expected the computed orientation pretty much depends on the order of the used 


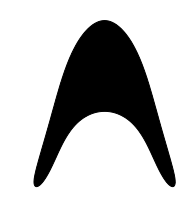

(a)

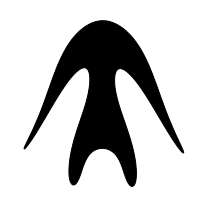

(b)

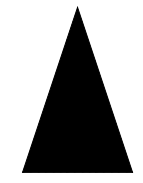

(c)

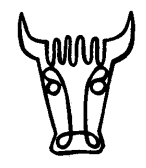

$(\mathbf{d})$

Figure 5: The presented figures have exactly one axis of symmetry.

Table 2: The computed orientations (for the shapes from Fig.5) show that the result do not depend much on the degree of $I_{N}$.

\begin{tabular}{lcccc}
\hline \hline & Fig. 5(a) & Fig. 5(b) & Fig. 5(c) & Fig. 5(d) \\
\hline$N=2$ & $\approx 90^{\circ}$ & $\approx 90^{\circ}$ & $\approx 90^{\circ}$ & $\approx 90^{\circ}$ \\
\hline$N=4$ & $\approx 90^{\circ}$ & $\approx 90^{\circ}$ & $\approx 90^{\circ}$ & $\approx 94^{\circ}$ \\
\hline$N=6$ & $\approx 90^{\circ}$ & $\approx 90^{\circ}$ & $\approx 90^{\circ}$ & $\approx 92^{\circ}$ \\
\hline$N=8$ & $\approx 90^{\circ}$ & $\approx 90^{\circ}$ & $\approx 90^{\circ}$ & $\approx 93^{\circ}$ \\
\hline$N=10$ & $\approx 90^{\circ}$ & $\approx 90^{\circ}$ & $\approx 90^{\circ}$ & $\approx 92^{\circ}$ \\
\hline \hline
\end{tabular}

central moment of inertia in the cases of Fig.6(a), Fig.6(b), and Fig.6(c). It is perhaps surprising that the computed orientation of the shape on Fig.6(d) does not depend much on the degree if the $I_{N}$ used.

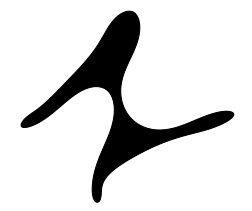

(a)

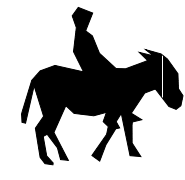

(b)

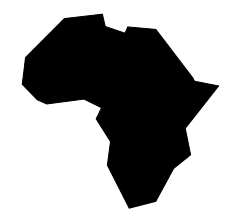

(c)

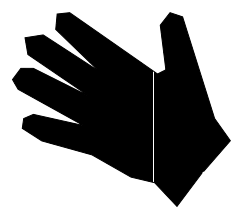

(d)

Figure 6: Non regular shapes.

The last group of examples consists of non-regular shapes whose orientation can be understood as well determined. Our expectation that orientation will not vary too much if we change the order of $I_{N}$ is confirmed by our experiments. Perhaps a so stable measured orientation is not expected 
Table 3: The computed orientation for the first three shapes from Fig. 6 pretty much depends on the used degree of the moment of inertia. In the forth case the orientation does not depend essentially on the used degree.

\begin{tabular}{lcccc}
\hline \hline & Fig. 6(a) & Fig. 6(b) & Fig. 6(c) & Fig. 6(d) \\
\hline$N=2$ & $\approx 48^{\circ}$ & $\approx 114^{\circ}$ & $\approx 153^{\circ}$ & $\approx 133^{\circ}$ \\
\hline$N=4$ & $\approx 56^{\circ}$ & $\approx 131^{\circ}$ & $\approx 158^{\circ}$ & $\approx 133^{\circ}$ \\
\hline$N=6$ & $\approx 61^{\circ}$ & $\approx 32^{\circ}$ & $\approx 163^{\circ}$ & $\approx 133^{\circ}$ \\
\hline$N=8$ & $\approx 63^{\circ}$ & $\approx 31^{\circ}$ & $\approx 167^{\circ}$ & $\approx 133^{\circ}$ \\
\hline$N=10$ & $\approx 64^{\circ}$ & $\approx 31^{\circ}$ & $\approx 170^{\circ}$ & $\approx 134^{\circ}$ \\
\hline \hline
\end{tabular}

in the case of Fig.7(d): The typed statement of Theorem 1 is taken as an image. Visually, it can be said that the orientation should be close to $0^{\circ}$ but the effect of italic letters that are not orthogonal to the vertical line could be expected to be bigger.

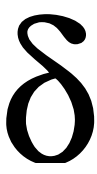

(a)

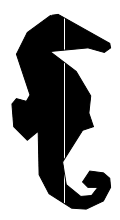

(b)

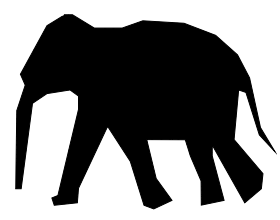

(c)
Theorem. For an n-fold rotationally symmetric shape having the centroid coincident with the origin, its $N$-th order central moment of inertia is constant about any line going through its centroid for all $N$ less than $n$.

(d)

Figure 7: Shapes whose orientation seems to be well-defined.

\section{Concluding Remarks}

In this paper we consider some problems related to the shape orientation. The most studied situation, where such problems arise, is when working with shapes having several axes of symmetry and when working with $n$-fold rotationally symmetric shapes. The paper is mainly based on the results presented in [11]. A very short proof of the main result from [11] is presented. Also, some disadvantages of the method for a use of higher order principal axes (from the same paper) are 
Table 4: Orientations of shapes from Fig. 7 as computed by applying Definition 2.

\begin{tabular}{lcccc}
\hline \hline & Fig. 7(a) & Fig. 7(b) & Fig. 7(c) & Fig. $7(\mathrm{~d})$ \\
\hline$N=2$ & $\approx 87^{\circ}$ & $\approx 96^{\circ}$ & $\approx 165^{\circ}$ & $\approx 0^{\circ}$ \\
\hline$N=4$ & $\approx 88^{\circ}$ & $\approx 95^{\circ}$ & $\approx 172^{\circ}$ & $\approx 1^{\circ}$ \\
\hline$N=6$ & $\approx 88^{\circ}$ & $\approx 94^{\circ}$ & $\approx 175^{\circ}$ & $\approx 0^{\circ}$ \\
\hline$N=8$ & $\approx 88^{\circ}$ & $\approx 93^{\circ}$ & $\approx 177^{\circ}$ & $\approx 0^{\circ}$ \\
\hline$N=10$ & $\approx 89^{\circ}$ & $\approx 92^{\circ}$ & $\approx 178^{\circ}$ & $\approx 0^{\circ}$ \\
\hline \hline
\end{tabular}

discussed. That lead to a suggestion that a slight modification to the method should be made.

It is clarified that the most of the problems come from the fact that the function (2) (used if the standard method is applied) is not complex enough to be successfully applied to all shapes. Since the shape orientation and shape elongation are problems that are considered together very often, let us just mention that problems related to the elongation computation come from similar reasons. In the standard approach, the ratio between $\max _{\delta \in[0,2 \pi)} I(\delta, S)$ and $\min _{\delta \in[0,2 \pi)} I(\delta, S)$

$$
\mathcal{E}(S)=\frac{\max \{I(\delta, S) \mid \delta \in[0,2 \pi]\}}{\min \{I(\delta, S) \mid \delta \in[0,2 \pi]\}}
$$

is used to estimate the elongation of $S$. If $I(\delta, S)=$ constant then the measured elongation equals 1 and it is minimum possible. The same measured elongation equal to 1 has a circle. That is not a desirable property if we would like to understand a circle as the least elongated shape. So, an alternative elongation measure could be useful if we would like to see, let say, a square as more elongated shape than the circle is. In accordance with the idea of [11] and discussion in this paper a higher order central moments can be used. So, we give the following definition.

Definition 3 Fix $N=2 k$. Then the elongation $\mathcal{E}_{N}(S)$ of a given shape $S$ is defined as

$$
\mathcal{E}_{N}(S)=\frac{\max \left\{I_{N}(\delta, S) \mid \delta \in[0,2 \pi]\right\}}{\min \left\{I_{N}(\delta, S) \mid \delta \in[0,2 \pi]\right\}}
$$

Table 5 presents the results if the elongation is computed as the ratio of the minimum and maximum value of the central moments having the order 2, 4, 6, 8, and 10 for the shapes from Fig. $4-7$. 
Table 5: The elongation $\mathcal{E}_{N}$ of shapes from Fig. 4-7 measured in accordance with Definition 3 for different values of $N$.

\begin{tabular}{|c|c|c|c|c|}
\hline & Fig. 4(a) & Fig. 4(b) & Fig. 4(c) & Fig. 4(d) \\
\hline$N=2$ & 1.0088 & 1.0058 & 1.0195 & 1.0055 \\
\hline$N=4$ & 1.0157 & 1.0112 & 1.9057 & 1.0174 \\
\hline$N=6$ & 1.1703 & 1.0161 & 3.819 & 1.0304 \\
\hline$N=8$ & 1.4785 & 1.0208 & 7.6845 & 1.0432 \\
\hline \multirow[t]{2}{*}{$N=10$} & 1.9412 & 1.0283 & 15.4992 & 1.0618 \\
\hline & Fig. 5(a) & Fig. 5(b) & Fig. 5(c) & Fig. 5(d) \\
\hline$N=2$ & 1.2476 & 1.9887 & 2.9994 & 1.8390 \\
\hline$N=4$ & 2.2053 & 2.6497 & 8.9946 & 2.9433 \\
\hline$N=6$ & 5.2786 & 3.2510 & 30.9537 & 4.6274 \\
\hline$N=8$ & 13.6571 & 3.9350 & 116.6828 & 8.2032 \\
\hline \multirow[t]{2}{*}{$N=10$} & 36.0603 & 4.8886 & 457.1119 & 15.8163 \\
\hline & Fig. 6(a) & Fig. 6(b) & Fig. 6(c) & Fig. 6(d) \\
\hline$N=2$ & 1.4112 & 4.2725 & 2.3052 & 2.2092 \\
\hline$N=4$ & 1.9276 & 12.9791 & 4.6661 & 4.0092 \\
\hline$N=6$ & 3.9397 & 39.2807 & 9.5060 & 6.3202 \\
\hline$N=8$ & 9.5492 & 126.2137 & 20.5897 & 9.2563 \\
\hline \multirow[t]{2}{*}{$N=10$} & 23.4180 & 414.6660 & 46.9665 & 13.2834 \\
\hline & Fig. 7(a) & Fig. 7(b) & Fig. 7(c) & Fig. $7(\mathrm{~d})$ \\
\hline$N=2$ & 2.9669 & 6.1161 & 1.9685 & 3.7635 \\
\hline$N=4$ & 10.6323 & 31.4050 & 3.5661 & 14.9106 \\
\hline$N=6$ & 37.6521 & 142.8998 & 6.4204 & 61.0137 \\
\hline$N=8$ & 129.3034 & 624.00 & 11.6162 & 257.7485 \\
\hline$N=10$ & 433.5460 & 2687.9 & 21.3072 & 1131.0 \\
\hline
\end{tabular}




\section{References}

[1] B.K.P. Horn, Robot Vision, MIT Press, Cambridge, MA, 1986.

[2] R. Jain, R. Kasturi, B.G. Schunck, Machine Vision, McGraw-Hill, New York, 1995.

[3] R. Klette, J. Žunić, "Digital approximation of moments of convex regions," Graphical Models and Image Processing, Vol. 61, pp. 274-298, 1999.

[4] J.-C. Lin, W.-H. Tsai, J.-A. Chen, "Detecting Number of Folds by a Simple Mathematical Property," Patt. Rec. Letters, Vol. 15, pp. 1081-1088, 1994.

[5] J.-C. Lin, "The Family of Universal Axes," Patt. Rec., Vol. 29, pp. 477-485, 1996.

[6] E. Mach, The analysis of sensations (Beiträge zur Analyse der Empfindungen), Routledge, London, 1996.

[7] G. Marola, "On the Detection of Axes of Symmetry of Symmetric and Almost Symmetric Planar Images," IEEE Trans. PAMI, Vol. 11, No. 6, pp. 104-108, 1989.

[8] S.E. Palmer, Vision Science: Photons to Phenomenology, MIT Press, 1999.

[9] D. Shen, H. H. S. Ip, "Optimal Axes for Defining the Orientations of Shapes," Electronic Letters, Vol. 32, No. 20, pp. 1873-1874, 1996.

[10] D. Shen, H. H. S. Ip, K. K. T. Cheung, and E. K. Teoh, "Symmetry Detection by Generalized Complex (GC) Moments: A Close-Form Solution," IEEE Trans. PAMI, Vol. 21, No. 5, pp. 466-476, 1999.

[11] W.H. Tsai, S.L. Chou, "Detection of Generalized Principal Axes in Rotationally Symmetric Shapes," Patt. Rec., Vol. 24, pp. 95-104, 1991. 